\title{
Electrocardiogram Vendor Name
}

National Cancer Institute

\section{Source}

National Cancer Institute. Electrocardiogram Vendor Name. NCI Thesaurus. Code C83262.

The literal identifier of the vendor or laboratory that performs an electrocardiogram examination. 